\title{
Neurosurgical Shunt Treatment of Paediatric Hydrocephalus: Epidemiology and Influencing Factors on Revision Surgeries - a Single-centre Retrospective Analysis of 131 Patients
}

Jasmin Nagl ( $\sim$ Jasmin.Nagl@neuro.med.uni-giessen.de )

Universitätsklinikum Gießen und Marburg Standort Gießen: Universitatsklinikum Giessen und Marburg GmbH Standort Giessen https://orcid.org/0000-0002-8647-4033

Frank Patrick Schwarm

Justus-Liebig-University Giessen

Michael Bender

Justus-Liebig-University Giessen

Aylin Gencer

Justus-Liebig-University Giessen

Harald Ehrhardt

Justus-Liebig-University Giessen

Andreas Hahn

Justus-Liebig-University Giessen

Bernd A. Neubauer

Justus-Liebig-University Giessen

Malgorzata Kolodziej

Justus-Liebig-University Giessen

\section{Research Article}

Keywords: hydrocephalus, shunt complication, revision surgery, infection, shunt dysfunction, shunt malfunction

Posted Date: November 30th, 2021

DOI: https://doi.org/10.21203/rs.3.rs-1086892/v1

License: (9) This work is licensed under a Creative Commons Attribution 4.0 International License. Read Full License 


\section{Abstract}

Paediatric hydrocephalus is a result of cerebrospinal fluid circulatory dysfunction and has diverse pathogeneses. This study investigates the epidemiology of paediatric hydrocephalus and influences of primary aetiology, type of implants, and of complications to develope new therapeutic approaches and strategies. Between 2013-2018 a retrospective analysis of 131 children, suffering from hydrocephalus, was conducted. Medical charts, operative reports and clinical follow-up visits were reviewed. Statistical analysis was performed using t-test/ANOVA and Kruksal-Wallis-test/ Mann-Whitney-U test. Most common pathogeneses of hydrocephalus among our patients were meningomyelocele associated and posthaemorrhagic. The majority received a programmable differential pressure valve (PP valve, $77.8 \%$ ) or a fixed differential pressure valve with gravitational unit (FPG valve, 14.8\%). Among 333 shunt associated surgeries $66 \%$ were performed because of mechanical shunt dysfunction $(61 \%)$, infection $(12 \%)$, or due to other reasons $(27 \%)$. The median rate of revisions for each patient within one year was 0.15 (IQR ${ }^{25-}$ $75: 0.00-0.68)$ and was influenced by aetiology $(p=0.045)$ and used valves $(p=0.029)$. The highest rates were seen in patients with posthaemorrhagic hydrocephalus and in those with FPG valve, the lowest in meningomyelocele associated hydrocephalus and PP valve. The occurrence of mechanical dysfunctions showed a relation to FPG valve $(\mathrm{p}=0.014)$. Furthermore, the median time interval between initial shunt surgery and infections was shorter than in mechanical dysfunctions $(p=0.033)$.

Conclusion: We could state several influence factors on revision surgeries in paediatric shunt patients. With the assessment of patients' risk profiles, we can classify paediatric shunt patients and could avoid unnecessary examinations or invasive procedures. Furthermore, we could prevent revisions surgeries, if we choose shunt material in accordance with the patients' associated shunt complications.

\section{Introduction}

The prevalence of hydrocephalus is reported at 88 cases in 100.000 children (age $\leq 18)[7,14]$. The pathogenesis is a cerebrospinal fluid (CSF) circulatory dysfunction. The mismatch of production and absorption of CSF leads to neurological symptoms. [6].

The aetiology of this dysfunction is diverse. Many cases occur immediately after birth due to dysfunction of CSF absorption (malresorptive hydrocephalus). Congenital hydrocephalus is often associated with the syndromic incidence of a meningomyelocele. Another cause of malresorptive hydrocephalus is related to perinatal intraventricular hemorrhage. In this pathology, absorption of CSF is dysfunctional due to high protein content or due to destruction of arachnoid granulations. Occlusive hydrocephalus, as in aquaeduct stenosis or tumour blockage, is rare [18].

The most common treatment for hydrocephalus is ventriculoperitoneal (VP) shunting, followed by ventriculoatrial and ventriculopleural shunting. [8]. The valves used mostly in our study were programmable differential pressure valves (PPV) or fixed differential pressure valves with gravitational unit (FPgV). The underlying mechanism of PPV is a ball-spring-mechanism. When CSF pressure reaches 
the opening pressure, the ball moves and opens the valve, allowing CSF to drain. The spring is attached to a rotating spiral, which controls the resistance of the spring towards the ball and can be adjusted by non-invasive methods using a codified magnetic field (opening pressure:30 $\mathrm{mmH}_{2} \mathrm{O}-200 \mathrm{mmH}_{2} \mathrm{O}$ )[21]

The major difference of FPG and PPV is the combination of a ball-in-cone mechanism and a gravitational unit. The ball-cone unit controls the CSF drainage analogous to the ball-spring-mechanism in a horizontal position and, additionally, a second unit controls the CSF drainage in a vertical position to prevent overdrainage when changing posture. The FPgV has fixed pressure levels [10]. Besides different mechanisms of the valve, companies set different priorities. Some focus on the adjustability of the CSF drain level or on the development of minor valves for infants [21]. Others focus on valves with high flow velocity to counteract obstruction of the valve or to achieve steadiness to environmental influences and medical examinations, e.g. magnetic resonance imaging [10].

Although the insertion of a VP-shunt system is one of the most common neurosurgical procedures, dysfunction or infection of the shunt system are frequent. Every second patient undergoes at least one additional surgery within the first three years after initial implantation because of shunt failure [25]. 3-15 $\%$ of paediatric patients suffer from an infection of the shunt system. If this occurs, the whole shunt system has to be removed. After antibiotic treatment a new system has to be implanted. Another frequent cause of shunt failure occurs due to occlusion of the valve or a dislocation of the ventricular/peritoneal catheter. The malfunctioning part will be surgically removed and exchanged.

Overall, an indwelling shunt system implies a high risk for surgeries and longer in-patient stays causing a limitation of quality of life [17]. Presently, there are no established guidelines as to which shunt system should be used in the presence of which pathology of hydrocephalus, nor as to the specific requirements of shunt systems over the different pathologies.

Therefore, the aim of this study was to investigate the influence of pathogenesis and shunt materials on incidence and type of complications, in order to establish a better understanding of and better treatment for paediatric hydrocephalus $(\mathrm{PH})$ and, additionally, to reduce complications of the shunt treatment in $\mathrm{PH}$ patients.

\section{Materials And Methods}

Between 2013 und 2018 a retrospective analysis of 131 children (64 female, 67 male) was conducted. All participants underwent shunt surgery in our institution and were 17 years old or younger at the time of primary shunt surgery. This study was approved by the institutional research ethics board (AZ165/14) and is based on medical charts, operative reports and clinical follow-up documentations. The determined parameters were age at shunt implantation, diagnosis, type of shunt, used catheters and valves, revision surgeries, type of revision, and reason for revision and were assembled anonymous. The database was created with Excel (Microsoft ${ }^{\circledR}$, USA) and the statistical analysis was conducted with SPSS Statistics 24 (IBM corp, USA). Normal distribution was tested by Kolmogorov-Smirnov test. Differences of two or more cohorts were tested using t-test and ANOVA as parametric tests. As non-parametric tests, Kruskal-Wallis- 
Test and Mann-Whitney-U- test were used. Chi-square test was used to evaluate the distribution within a cohort or between two patient cohorts. A p-value $<0.05$ was considered statistically significant.

\section{Results}

\section{Epidemiology of patients}

The most common aetiology in our cohort was the malresorptive hydrocephalus. MMCaH occurred in $35.1 \%$, $\mathrm{PPH}$ in $24.4 \%$, and congenital non-meningomyelocele-associated in $9.2 \%$ of patients. Hydrocephalus occlusus caused by stenosis was found in $8.4 \%$ of patients and by tumour in $7.6 \%$ of patients. The remaining patients $(15.3 \%)$ showed rare underlying pathomechanisms.

Median age of patients undergoing their first shunt surgery was 35 days (IQR ${ }^{25-75}: 17-170$ days). The largest group (48.5\%) was in their first month of life. While paediatric patients with MMCaH were at a median age of 0 months (IQR ${ }^{25-75}: 0-1$ month), patients with occlusive hydrocephalus caused by tumour were significantly older with a median age of 51 months (IQR $25-75: 8-106$ months); $\left(p<0.001^{\star \star \star}\right)$ (figure 1$)$.

Ventriculoperitoneal shunting was performed in $85 \%$. The most used valve was PPV (77.8\%), followed by FPgV (14.8\%). The remaining $7.4 \%$ of patients received an uncommon valve or did not have one.

\section{Revision surgeries}

In our study 333 hydrocephalus associated surgeries were performed. 66\% $(n=220)$ were revision surgeries, $34 \%$ were initial shunt surgeries. The median follow-up time per patient was 41.5 months (IQR ${ }^{25-75}: 21.0-57.5$ months). In the cohort of 112 patients, whose follow-up was completely at our institution and was fully documented, 62 patients (55\%) received revision surgery. In the cohort of 112 patients, whose follow-up was complete, 62 patients (55\%) received revision surgery. $50 \%$ of all patients were revised within the first year.

To evaluate the characteristics of revision surgery, the cause of revision was analyzed in detail. $61 \%$ of surgeries were performed because of mechanical dysfunction. In $63 \%$ of cases the valve was changed, making it the most revised part of the shunt system. Ventricular and peritoneal catheters were changed in $56 \%$ and $36 \%$, respectively. $12 \%$ were performed because of infection of the shunt system, requiring a complete removal. Staphylococcus epidermidis was the most common pathogen (42.3\%), followed by enterococcus faecialis (7.7\%). In $34.6 \%$ no pathogen could be found. Other proven pathogens, each occurring once, were streptococcus pyogenes, escherichia coli, staphylococcus capitis and streptococcus pneumoniae.

Furthermore, the number of revisions within one year of indwelling shunt system (revisions/p/y) and the time interval to first revision were inspected. In a follow-up of at least one year, the median number of revisions/p/y was $0.15\left(\mathrm{IQR}^{25-75}: 0-0.68\right)$. The lowest rate of revisions/p/y was 0 , the highest 3 . Time range from initial shunt surgery to first revision surgery varied from 0 to 76 months. Thereby, an 
accumulation of low time interval to initial surgery was seen (figure 2$) ;\left(p<0.001^{\star \star \star}\right)$. The median interval was 1 month (IQR ${ }^{25-75}: 0-2$ months), meaning $38 \%$ of revision surgeries were performed one month after initial surgery.

\section{Influences on rate of revisions}

As a major influencing factor of the number of revisions $/ \mathrm{p} / \mathrm{y}$, the aetiologies of hydrocephalus were identified $\left(p=0.045^{\star}\right)$. While the median rate of revisions $/ \mathrm{p} / \mathrm{y}$ in $\mathrm{PPH}$ was $0.52\left(\mathrm{IQR}^{25-75}: 0.36-0.74\right)$, $\mathrm{MMCaH}$ showed the lowest rate with median $0\left(\operatorname{IQR}^{25-75}: 0-0.20\right)$;(figure 3).

The valve used at initial shunt surgery was another significant influencing factor $\left(p=0,029^{\star}\right)$ (figure 4 ). The median rate of revisions/p/y was $0\left(\mathrm{IQR}^{25-75}: 0-0.69\right)$ with the PPV (77.8\%) and $0.43\left(\mathrm{IQR}^{25-75}: 0.26\right.$ 0.66) with the FPgV (figure 4).

\section{Influences on cause of revision}

Aetiology of hydrocephalus did not only affect revision rate but had significant influence on the reason behind the revision $\left(p=0,040^{\star}\right)$. Patients with PPH had a significantly higher number of revisions caused by mechanical dysfunction than patients with $\mathrm{MMCaH}$ (figure 5). No significant difference could be found among infections that occurred with different aetiologies.

The implanted shunt valve was another remarkable factor influencing the onset of revision surgeries caused by mechanical dysfunction $\left(p=0,001^{\star *}\right)$. The median rate of mechanical dysfunctions in PPV was $0\left(\mathrm{IQR}^{25-75}: 0-0.185\right)$ and with FPgV 0,2 (IQR $\left.25-75: 0,10-0,31\right)$;(figure 6).

Furthermore, the cause of revision was associated with the time interval of initial and revision surgery $\left(p=0,002^{* *}\right)$;(figure 7). The median interval of the occurrence of infections was shorter (median 0 months $\left(\mathrm{IQR}^{25-75}: 0-1\right.$ months)) than the interval of the occurrence of mechanical dysfunctions (median 2.5 months (IQB ${ }^{25-75}: 1,0-11,0$ months). All infections occurred within 6 months after primary shunt surgery.

\section{Influences on time of revision}

Age at initial surgery was identified as an influencing factor on the time interval between initial and revision surgery $(\mathrm{p}<0,001)$ (figure 8 ). Patients, who received their shunt system within the first month of life had the shortest time interval to revision surgery (median 0 months (IQR ${ }^{25-75}: 0-0.75$ months)). Surgery after the third month entended the interval to 9 months (IQR ${ }^{25-75}: 4-19$ months).

\section{Discussion}

\section{The paediatric shunt patient}

The pathogenesis of hydrocephalus is diverse and could be classified in different groups $[4,18,22]$. In our cohort the MMCaH was most frequent, followed by PPH. Overall, our cohort represents the most common 
aetiologies of hydrocephalus in accordance with the current literature [11, 14]. Furthermore, the most frequent form of treatment in our cohort was VP-shunting [20].

A parameter which is not specifically described in $\mathrm{PH}$ patients is the distribution of age. In this study $48.5 \%$ of patients were newborns at the time of initial shunt implantation. Two thirds were in their first two months of life. In addition, patients with MMCaH or PPH were significantly younger than patients with occlusive hydrocephalus. Even though age distribution of hydrocephalus was analyzed in metaanalysis studies. The only statement was, that patients younger than 18 years had a significantly higher incidence of hydrocephalus than middle-aged patients [14, 26].

Both findings could be quite helpful to acquire a better understanding of the pathogenesis of $\mathrm{PH}$.

\section{Rate of revisions}

The treatment of $\mathrm{PH}$ is associated with a high number of complications despite the advances in technology and surgical technique [2]. In our cohort, an overall of $55 \%$ of patients were revised, $50 \%$ within the first year after receiving a shunt system. In the current literature, the rate of revisions within one year after surgery ranges from $25 \%$ [17] to $50 \%$ [11]. Reddy et al. (2014) showed a difference in paediatric and adult patients. In a median follow up of 6.5 years, $78.2 \%$ of paediatric patients underwent revision surgery, compared to $32.5 \%$ of adult patients [20]. In our study the high number of revisions is in accordance with the current literature and identifies paediatric patients, in contrast to adult patients, as high-risk patients.

We identified further factors as high risk for complications within the paediatric group. In our cohort, the younger the patients were at initial surgery, the more frequent reoperations had to be performed. In accordance with Gurbuz et al., who found an association between age at shunt implantation and the occurrence of infections, we concluded, the youngest shunt patients have the highest risk for revision surgeries [12].

In our cohort, $38 \%$ of revision surgeries were performed within the first month after initial surgery. Alatas et al. and Dupepe et al. reported an accumulation of revision surgeries within one year after shunt implantation in patients with $\mathrm{MMCaH}[1,9]$. Wang et al. concluded that patients with PPH had more revisions in the first three years than in the time thereafter [28]. These results suggest that the first month after shunt implantation is the most vulnerable period for the shunt system.

Aetiology was associated with the rate of revisions in our cohort. MMCaH occurred with low revision rates and the PPH with high revision rates. Reddy et al. analyzed, among other parameters, the aetiology as an influencing factor and described a high rate of revisions in $\mathrm{MMCaH}$ and a comparatively low rate in $\mathrm{PPH}$ [20]. The difference to our cohort was once again the patients' age. Their cohort consisted of paediatric and adult patients, which implicates that aetiology has an impact on paediatric and adult patients, but in different ways. Al-Hakim et al. showed in paediatric patients cohorts a high shunt survival rate in patients with MMCaH compared to other aetiologies, which was in accordance with our paediatric patients [3]. 
Furthermore, they see regular follow-up examinations as the reason for the low rate of revisions in MMC. In addition, MMC patients underwent surgical coverage of skin defect, which is accompanied by antibiotic therapy to avoid wound infections. This factor could further explain the low rate of revisions, shunt infections, and could not only help to calculate the risk for necessary surgical treatment for patients on the basis of pathogenesis of hydrocephalus in addition to age and time of indwelling shunt system, but also help to identify approaches for improvement of shunt treatment.

Kestle et al. provided a review about $\mathrm{PH}$ and emphasized the lack of current studies comparing different valves [15]. Therefore, in our study we compared the different shunt valves with regard to different mechanisms. We could show that the PPV was associated with a lower rate of revision and the FPgV with a higher rate of revision. Tervonen et al. analyzed the difference of revision rates in programmable and non-programmable valves but could not elaborate any significant difference [25]. Alavi et al. showed similar results without any significant difference in valves with and without gravitational units [2]. In summary, the differences we uncovered seem to be multifactorial. Because of its small size, the PPV was often used in patients with $\mathrm{MMCaH}$ which had the lowest rate of revisions in our cohort. Similarly, the valve implanted mostly in $\mathrm{PPH}$ was the FPgV, which had the highest revision rate.

For this reason, we evaluated the influences of shunt valves as well as age and aetiology on occurrence of shunt infections and mechanical dysfunctions in detail.

\section{Shunt infections}

Revision surgeries caused by infection occurred in $12 \%$. Malluci et al. detected infections in $12 \%$ within their paediatric cohort, Reddy et al. $21 \%[17,19]$. Similar to our cohort, the bacterial spectrum consisted mostly of staphylococcus epidermidis and staphylococcus aureus [17]. In our patient cohort we could show a significant shorter time between initial surgery and the occurrence of shunt infections than the occurrence of a mechanical dysfunction. Overall time until revision was analyzed in several studies with specific patient cohorts, but not the influence on cause for revision, especially on the occurrence of infections $[9,12]$.

The infantile immune system of young hydrocephalus patients and the bacterial spectrum indicate that shunt surgery itself is a risk factor and that there is need for improvement to avoid infections of the shunt system.

The chiefly evaluated shunt part in this study the valve, does not seem to be the vulnerable part of the shunt for infections. Malucci et al. determined the catheter as the main origin for shunt infections and showed the superiority of the antibiotic catheters [17]. However, in our cohort the shunt valve seems to be more consequential for the occurrence of mechanical dysfunctions.

\section{Mechanical dysfunctions}

In our cohort, revision surgeries caused by mechanical dysfunction occurred in $61 \%$. In contrast the current literature describes the majority of revisions by mechanical dysfunctions $[13,16,17,19]$. Malluci 
et al. mentioned, that revisions in $81 \%$ of patients were not caused by infections, but provided no further analysis [17]. The most revised part of shunt in our cohort was the valve (41\%), followed by the proximal (36\%) and distal (23\%) catheter. Browd et al. described obstruction of proximal and distal catheters as the primary reason for shunt revision [5]. Other studies confirmed these findings [13, 24, 27]. Hanak et al. described inflammatory reactions to the shunt catheters, which caused obstruction in a varying time interval after initial surgery. As other possible causes for mechanical dysfunction they described catheter length and the decreasing ability of the peritoneum to absorb CSF, e.g. after abdominal surgeries [13]. These outlined causes are not associated with young age. Therefore, distal catheter obstruction seems to be a problem in adult but not in paediatric patients. As a result, the predominantly revised part in our study cohort, the valve, appears to be the most fragile part of the shunt system for mechanical dysfunctions in paediatric patients and presents a starting point in the combat to avoid shunt revisions.

While the valve did not have any significant influence on the occurrence of infections, we showed an association between the valve and the occurrence of mechanical dysfunctions. The FPgV was associated with significantly more mechanical dysfunctions than the PPV. Analogous to the higher revision rate in general, the FPgV was often used in patients with PPH, which was associated with a higher revision rate. If this was the only reason for the higher rate of mechanical dysfunctions, infections should have also been higher in FPgV, which did not occur. Therefore, the underlying reason seems to be the valve itself. An argument could be the technical details of the valves. The CSF must pass through two mechanisms in FPgVs. If the CSF has high protein levels, obstruction is more likely to occur and subsequently to require surgical treatment $[10,13]$. Additionally the vulnerability of valves could be increased by more sensitive components and the valve could be more vulnerable for minor traumas or a MRI examination [23].

The same mechanism, obstructions caused by high protein levels in CSF, seems to be responsible for the influence of aetiology on the occurrence of mechanical dysfunctions. The highest rate of mechanical dysfunctions was seen in $\mathrm{PPH}$. In contrast, $\mathrm{MMCaH}$ had a different rate of infection occurrence and mechanical obstructions, which leaned more heavily in favor of infections compared to PPH. Alatas et al. showed the same findings in 31 patients with $\mathrm{MCCaH}$, where $43 \%$ got revision surgery due to infection and $14 \%$ due to mechanical dysfunction [1]. These results provide a novel approach in the improvement of shunt treatment and a step forward in realizing individually adaptable shunt treatment.

A limitation of this study was its retrospective character. Another aspect was the limited number of patients. Even though a randomized trial could not be conducted, our study cohort could still be seen as a representative cohort of $\mathrm{PH}$ patients.

\section{Conclusion}

According to the assessment of the individual risk profiles of patients, the shunt material should be chosen individually. To prevent formidable shunt infection in patients with $\mathrm{MMCaH}$, we could perform preventive antibiotic therapy even before surgery. We could help in preventing frequent obstruction of valves in PPH patients if we use a valve, which is associated with a lower rate of mechanical 
dysfunctions, e.g. a valve with only one unit. A regular follow-up examination plan should be provided to improve the timely recognition of shunt complications. Further prospective, multi-center studies are required to help in preventing revision surgeries.

\section{Declarations}

Funding: Authors state no funding involved.

Conflict of interest/Competing interests: Authors declared no conflicts of interest.

Availability of data and material: All data was collected in connection with the treatment of the patients, independently of this study. The study data was collected retrospectively and were assembled anonymous.

Code availability: N/A

Ethics approval: Approved by the institutional research ethics board (10/2019, AZ 165/14). The research related to human use complies with all the relevant national regulations, institutional policies and was performed in accordance with the tenets of the Helsinki Declaration, and has been approved by the authors' institutional review board or equivalent committee.

Consent to participate: This study does not contain any studies with human participants. The study data was collected retrospectively and were assembled anonymous.

Consent to publish: All contributed authors declared consent for publication.

Author contributions: Conceptualization: M. Kolodziej; Methodology, Formal analysis and investigation: J. Nagl; Writing - original draft preparation: J. Nagl; Writing - review and editing: M. Kolodziej, F. P. Schwarm, A. Gencer, M.Bender; Resources: H. Erhardt, A. Hahn, B.A. Neubauer; Supervision: M. Kolodziej.

\section{References}

1. Alatas I, Canaz G, Kayran NA et al. (2018) Shunt revision rates in myelomeningocele patients in the first year of life: a retrospective study of 52 patients. Childs Nerv Syst 34:919-923. doi: 10.1007/s00381-017-3663-z

2. Alavi S, Schulz M, Schaumann A et al. (2017) Valve exchange towards an adjustable differential pressure valve with gravitational unit, clinical outcome of a single-center study. Childs Nerv Syst 33:759-765. doi: 10.1007/s00381-017-3387-0

3. Al-Hakim S, Schaumann A, Schneider J et al. (2018) Experience in shunt management on revision free survival in infants with myelomeningocele. Childs Nerv Syst 34:1375-1382. doi:

$10.1007 /$ s00381-018-3781-2 
4. Amacher AL, Wellington J (1984) Infantile hydrocephalus: long-term results of surgical therapy. Childs Brain 11:217-229. doi: 10.1159/000120180

5. Browd SR, Ragel BT, Gottfried ON et al. (2006) Failure of cerebrospinal fluid shunts: part l: Obstruction and mechanical failure. Pediatr Neurol 34:83-92. doi:

10.1016/j.pediatrneurol.2005.05.020

6. Cserr HF (1971) Physiology of the choroid plexus. Physiol Rev 51:273-311. doi: 10.1152/physrev.1971.51.2.273

7. Dewan MC, Rattani A, Mekary R et al. (2018) Global hydrocephalus epidemiology and incidence: systematic review and meta-analysis. J Neurosurg:1-15. doi: 10.3171/2017.10.JNS17439

8. Di Rocco C, Massimi L, Tamburrini G (2006) Shunts vs endoscopic third ventriculostomy in infants: are there different types and/or rates of complications? A review. Childs Nerv Syst 22:1573-1589. doi: 10.1007/s00381-006-0194-4

9. Dupepe EB, Hopson B, Johnston JM et al. (2016) Rate of shunt revision as a function of age in patients with shunted hydrocephalus due to myelomeningocele. Neurosurg Focus 41:E6. doi: 10.3171/2016.8.FOCUS16257

10. Eymann R, Steudel W-I, Kiefer M (2007) Pediatric gravitational shunts: initial results from a prospective study. J Neurosurg 106:179-184. doi: 10.3171/ped.2007.106.3.179

11. Fernández-Méndez R, Richards HK, Seeley HM et al. (2019) Current epidemiology of cerebrospinal fluid shunt surgery in the UK and Ireland (2004-2013). J Neurol Neurosurg Psychiatry 90:747-754. doi: 10.1136/jnnp-2018-319927

12. Gurbuz MS, Yuksel MO (2020) The Association between the Timing of Shunt Placement and Shunt Infection in Hydrocephalus Associated with Myelomeningocele. Turk Neurosurg 30:94-98. doi: 10.5137/1019-5149.JTN.26510-19.1

13. Hanak BW, Bonow RH, Harris CA et al. (2017) Cerebrospinal Fluid Shunting Complications in Children. Pediatr Neurosurg 52:381-400. doi: 10.1159/000452840

14. Isaacs AM, Riva-Cambrin J, Yavin D et al. (2018) Age-specific global epidemiology of hydrocephalus: Systematic review, metanalysis and global birth surveillance. PLoS One 13:e0204926. doi: 10.1371/journal.pone.0204926

15. Kestle JRW, Riva-Cambrin J (2019) Prospective multicenter studies in pediatric hydrocephalus. J Neurosurg Pediatr 23:135-141. doi: 10.3171/2018.10.PEDS18328.

16. Kraemer MR, Sandoval-Garcia C, Bragg T et al. (2017) Shunt-dependent hydrocephalus: management style among members of the American Society of Pediatric Neurosurgeons. J Neurosurg Pediatr 20:216-224. doi: 10.3171/2017.2.PEDS16265

17. Mallucci CL, Jenkinson MD, Conroy EJ et al. (2019) Antibiotic or silver versus standard ventriculoperitoneal shunts (BASICS): a multicentre, single-blinded, randomised trial and economic evaluation. The Lancet 394:1530-1539. doi: 10.1016/S0140-6736(19)31603-4

18. McAllister JP (2012) Pathophysiology of congenital and neonatal hydrocephalus. Semin Fetal Neonatal Med 17:285-294. doi: 10.1016/j.siny.2012.06.004 
19. Reddy GK, Bollam P, Caldito G (2012) Ventriculoperitoneal shunt surgery and the risk of shunt infection in patients with hydrocephalus: long-term single institution experience. World Neurosurg 78:155-163. doi: 10.1016/j.wneu.2011.10.034

20. Reddy GK, Bollam P, Caldito G (2014) Long-term outcomes of ventriculoperitoneal shunt surgery in patients with hydrocephalus. World Neurosurg 81:404-410. doi: 10.1016/j.wneu.2013.01.096

21. Reinprecht A, Dietrich W, Bertalanffy A et al. (1997) The Medos Hakim programmable valve in the treatment of pediatric hydrocephalus. Childs Nerv Syst 13:588-93; discussion 593-4. doi: $10.1007 / \mathrm{s} 003810050145$

22. Rekate HL (2008) The definition and classification of hydrocephalus: a personal recommendation to stimulate debate. Cerebrospinal Fluid Res 5:2. doi: 10.1186/1743-8454-5-2

23. Schwarm FP, Nagl J, Bender M et al. (2020) Programmable valve breakage in shunt systems of children with posthemorrhagic hydrocephalus after minor head trauma-a case series. Childs Nerv Syst 36:2027-2031. doi: 10.1007/s00381-020-04541-6

24. Stone JJ, Walker CT, Jacobson M et al. (2013) Revision rate of pediatric ventriculoperitoneal shunts after 15 years. J Neurosurg Pediatr 11:15-19. doi: 10.3171/2012.9.PEDS1298

25. Tervonen J, Leinonen V, Jääskeläinen JE et al. (2017) Rate and Risk Factors for Shunt Revision in Pediatric Patients with Hydrocephalus-A Population-Based Study. World Neurosurg 101:615-622. doi: 10.1016/j.wneu.2017.02.030

26. Tully HM, Dobyns WB (2014) Infantile hydrocephalus: a review of epidemiology, classification and causes. Eur J Med Genet 57:359-368. doi: 10.1016/j.ejmg.2014.06.002

27. Turhan T, Ersahin Y, Dinc M et al. (2011) Cerebro-spinal fluid shunt revisions, importance of the symptoms and shunt structure. Turk Neurosurg 21:66-73

28. Wang JY, Jackson EM, Jallo GI et al. (2015) Shunt revision requirements after posthemorrhagic hydrocephalus of prematurity: insight into the time course of shunt dependency. Childs Nerv Syst 31:2123-2130. doi: 10.1007/s00381-015-2865-5

\section{Figures}




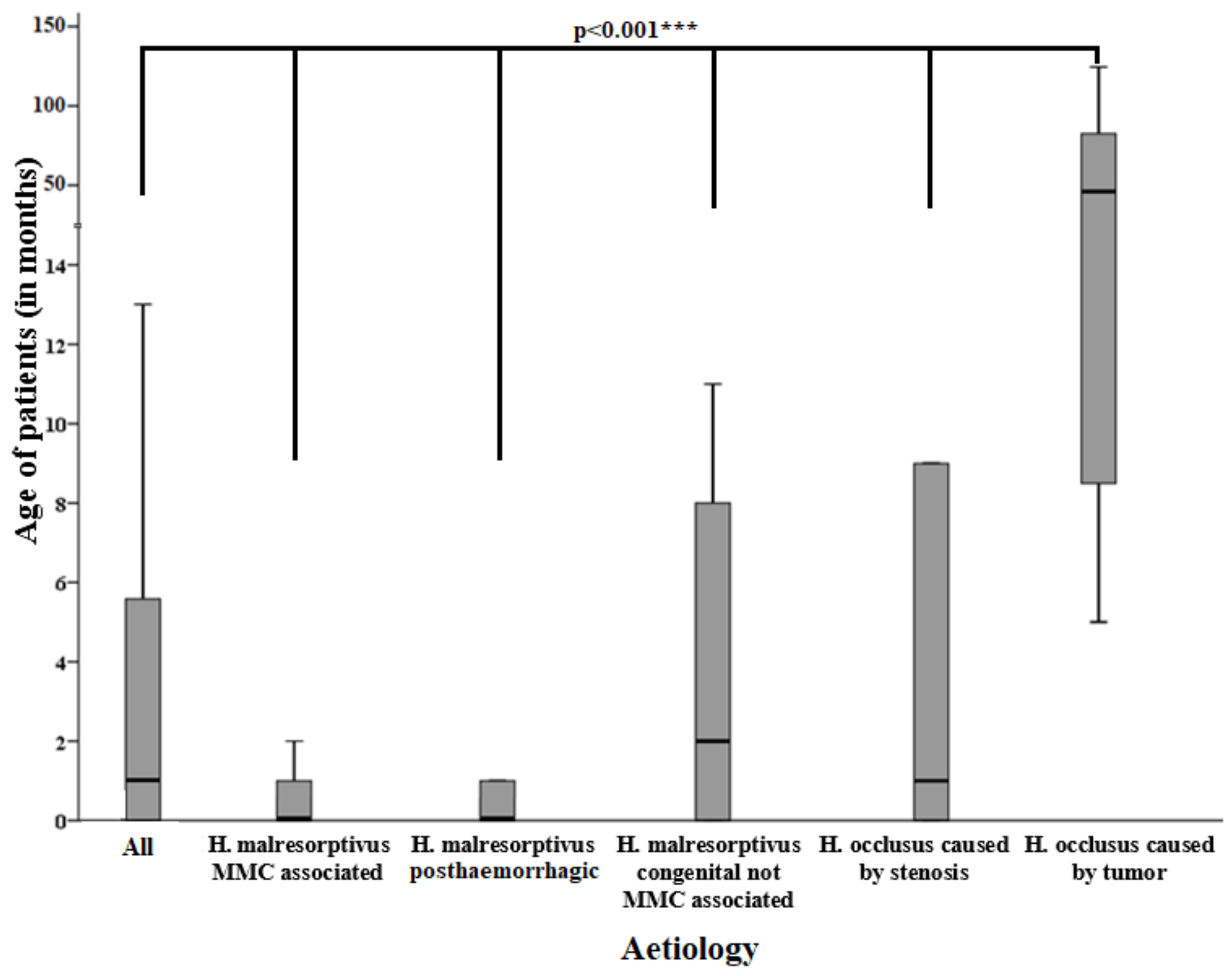

Figure 1

Box plot chart of age of patients overall and of pathomechanisms separated into different groups: The difference between the median ages of patients with different diagnoses was seen very clearly $\left(p<0.001^{\star \star *}\right)$ 


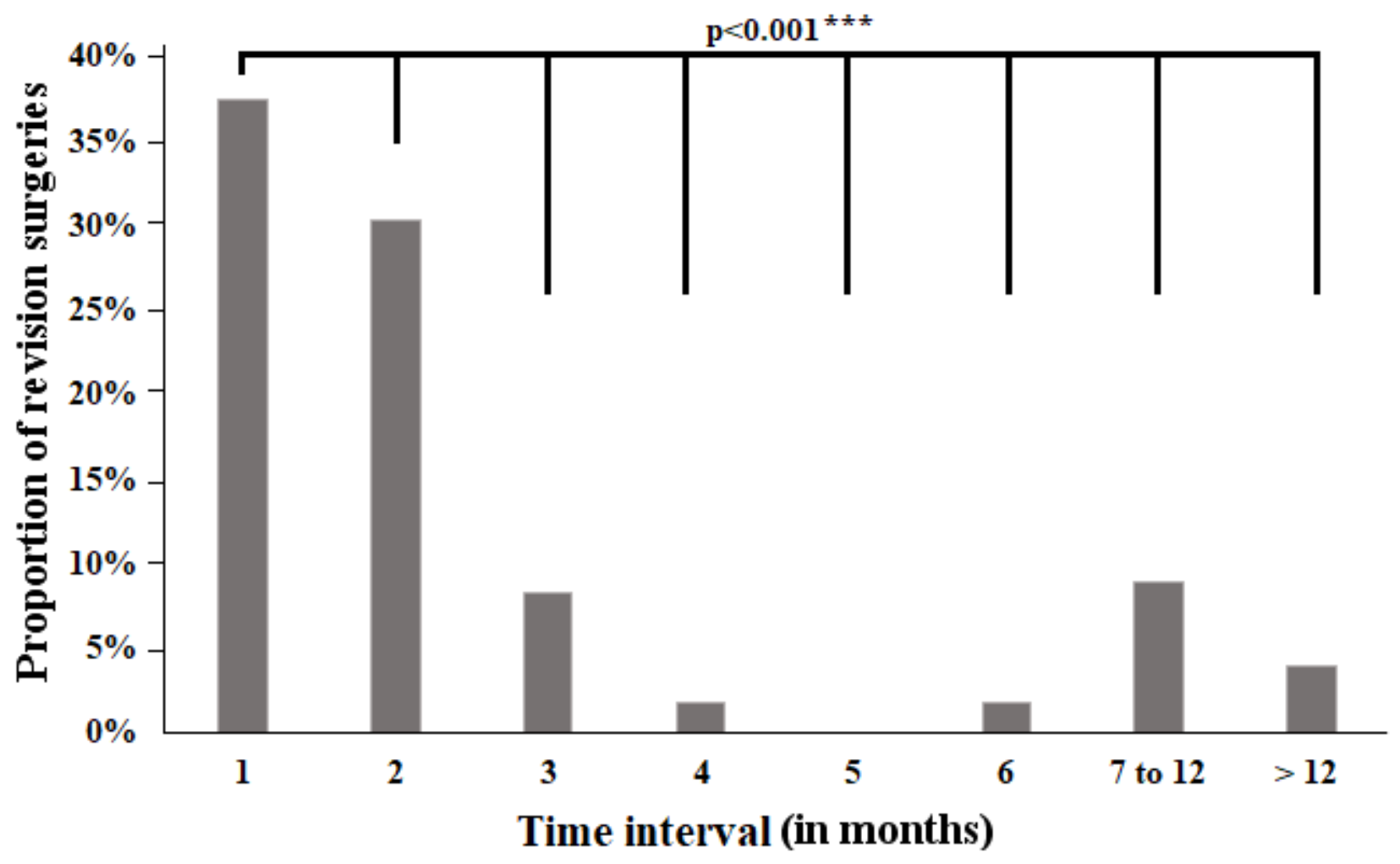

Figure 2

Bar graph of the proportion of revision surgeries grouped by time interval between initial shunt surgery and revision surgery: The largest amount of revision surgeries took place after only two months $\left(p<0.001^{\star \star \star}\right)$ 


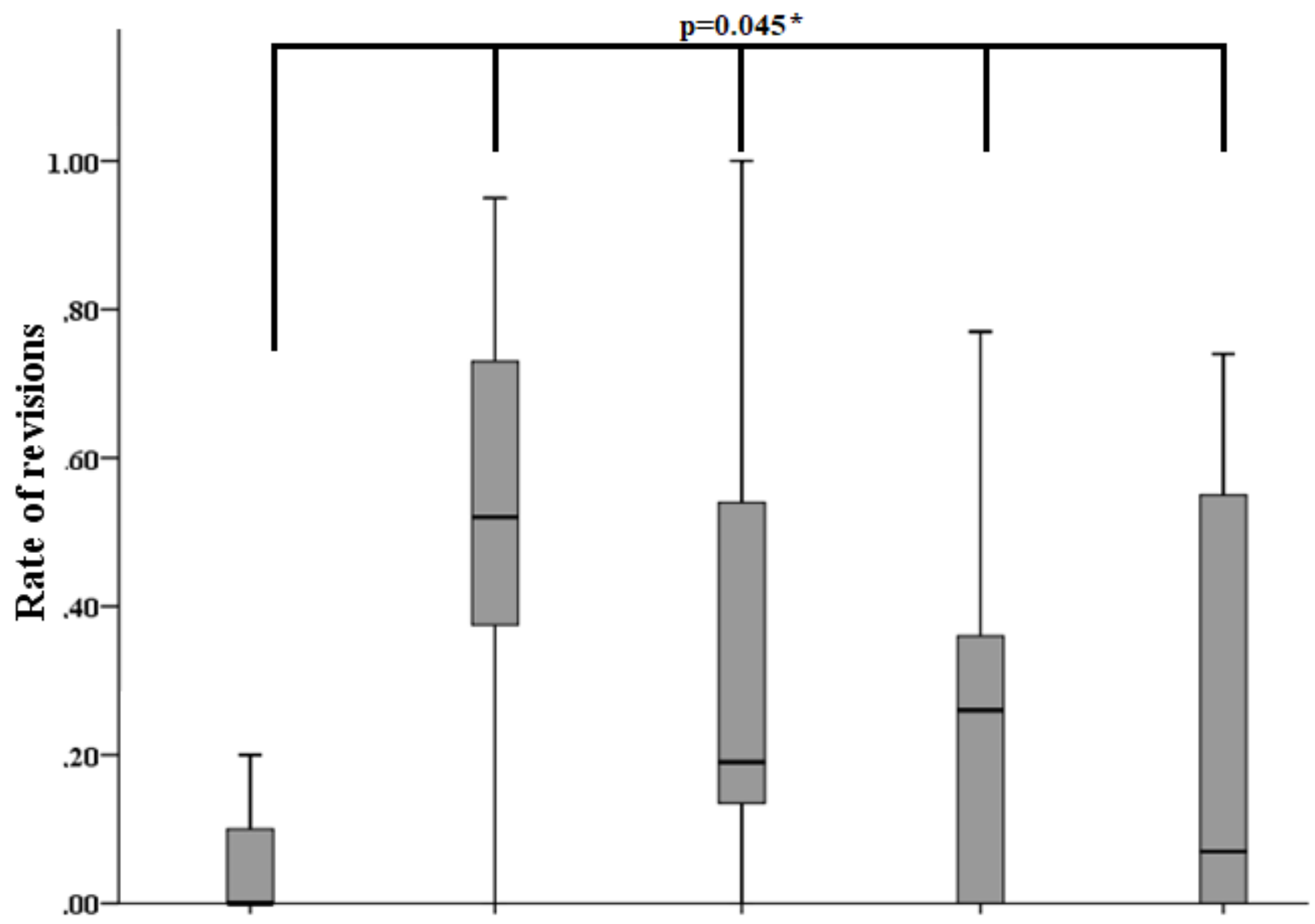

H. malresorptivus $H$. malresorptivus $H$. malresorptivus $H$. occlusus caused $H$. occlusus caused MMC associated posthaemorrhagic congenital not MMC associated by stenosis by tumor

\section{Aetiology}

Figure 3

Box plot graph of the influence of aetiology on the rate of revisions: The minor rate is seen in patients with $\mathrm{MMCaH}$, the major rate in patient with $\mathrm{PPH}\left(\mathrm{p}=0.045^{\star}\right)$ 


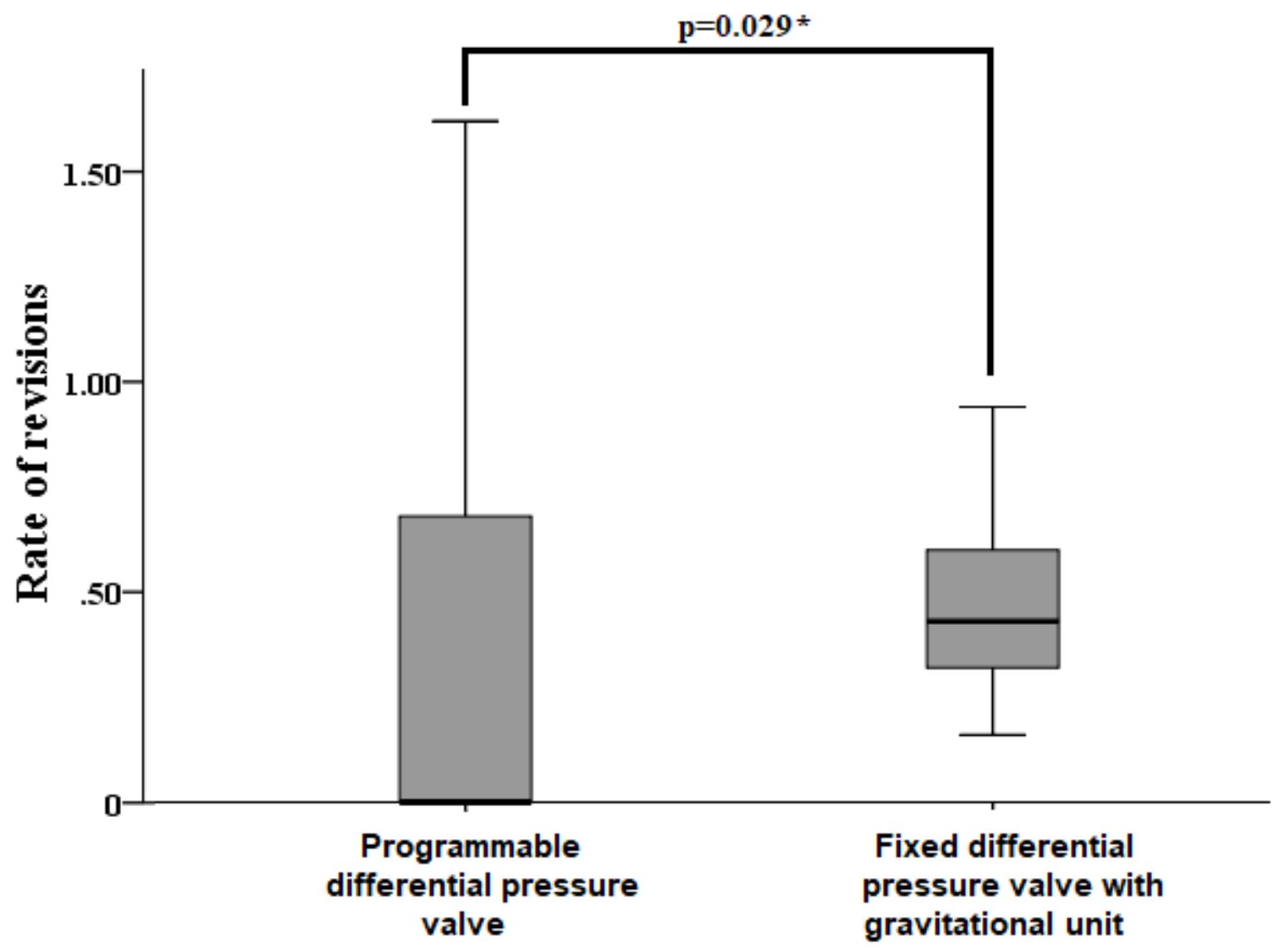

Figure 4

Box plot graph of the influence of the valve on the rate of revisions: The FPgV had a significantly higher rate of revisions than the PPV $\left(p=0,029^{*}\right)$ 


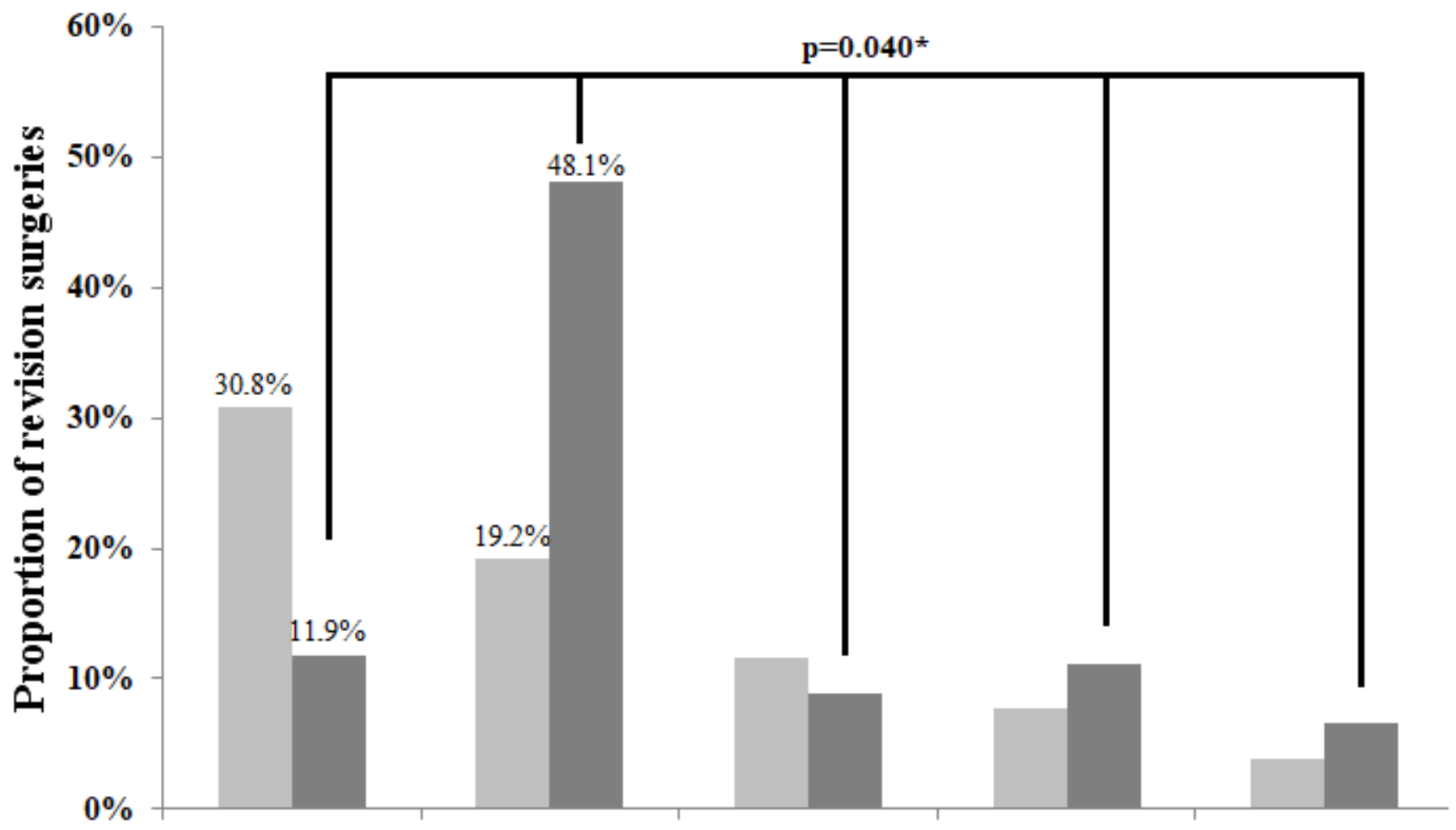

H. malresorptivus $H$. malresorptivus $H$. malresorptivus $H$. occlusus caused $H$. occlusus caused MMC associated posthaemorrhagic congenital not MMC associated by stenosis by tumor

\section{Aetiology}

\section{Infection}

Mechanical dysfunction

Figure 5

Influence of aetiology on the cause of revision pictured with a bar graph: The highest rate of mechanical dysfunctions was seen in PPH, the lowest rate in MMCaH $\left(p=0,040^{\star}\right)$ 


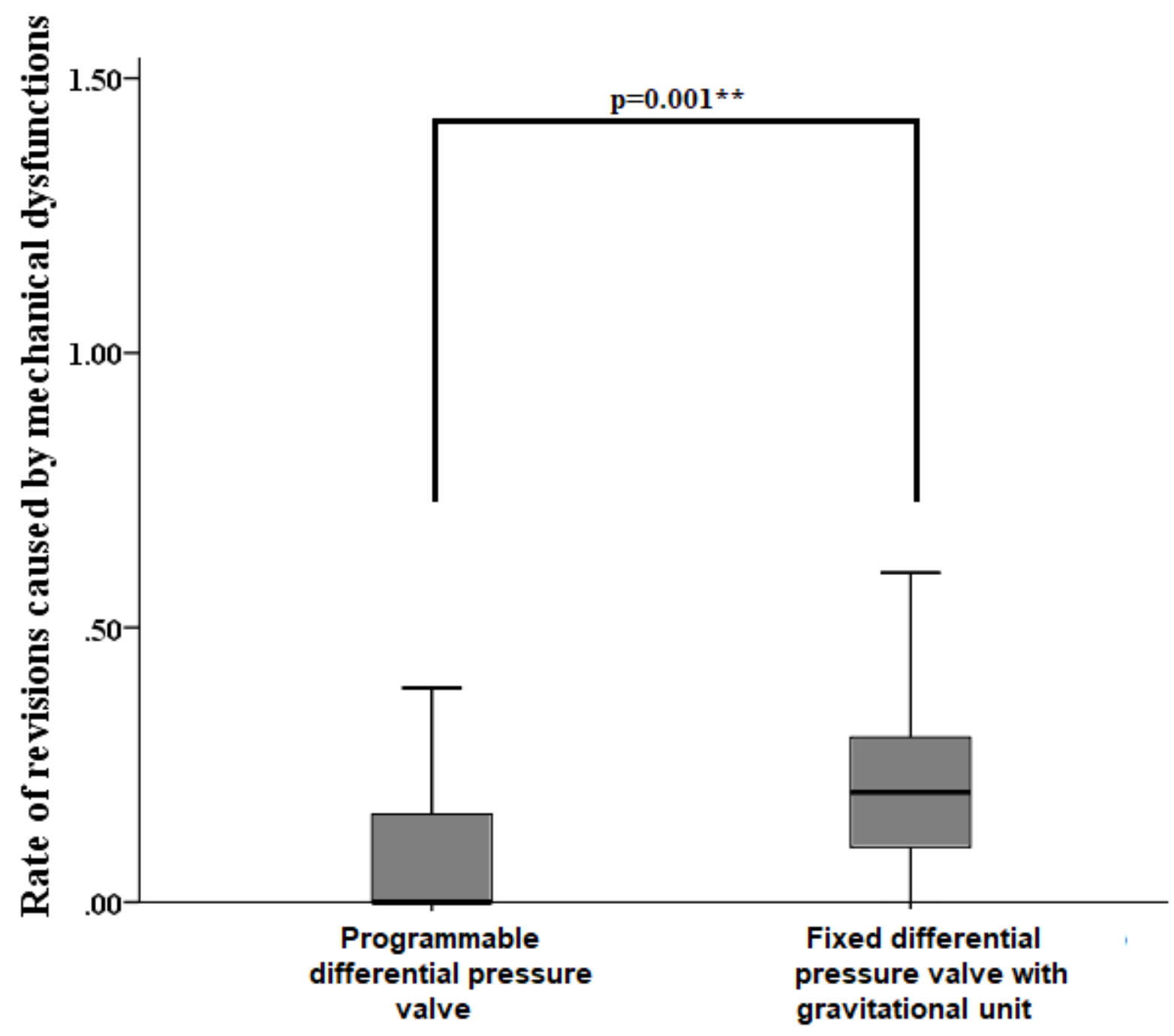

Figure 6

Box plot graph of the rate of revisions caused by mechanical dysfunctions, grouped by used valves: The FPgV had a significantly higher rate of revisions than the PPV $\left(p=0,001^{\star *}\right)$ 


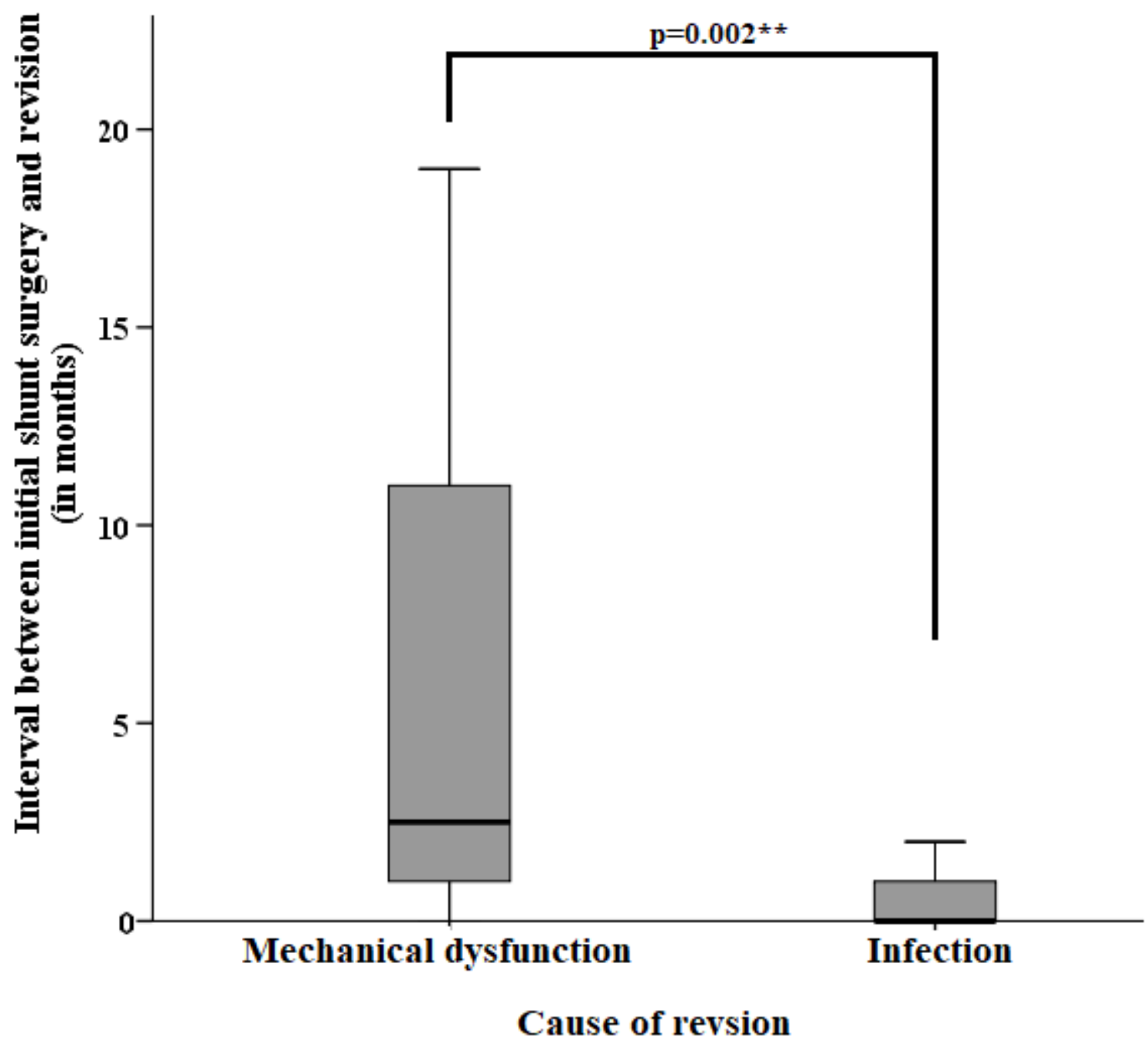

Figure 7

Box plot graph of the time interval of initial shunt surgery and revision surgery grouped by cause of revision: The interval in infections was significantly lower than in mechanical dysfunctions $(p=0,002 * \star)$ 


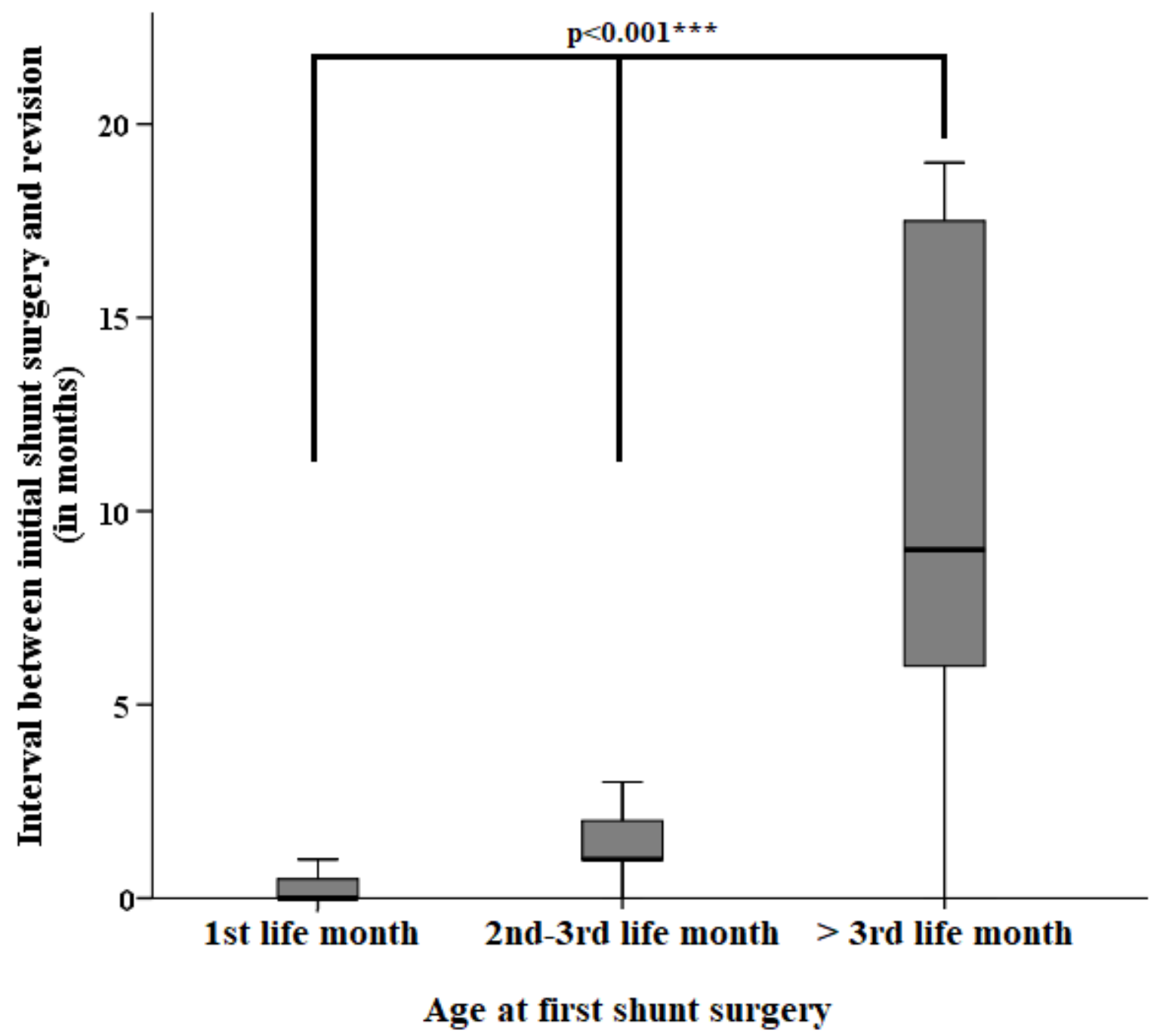

Figure 8

Box plot graph of the time interval between initial shunt surgery and revision surgery grouped by age at first shunt surgery: The interval gets longer as the age of patients at the time of surgery increases $\left(\mathrm{p}<0,001^{\star \star *}\right)$ 